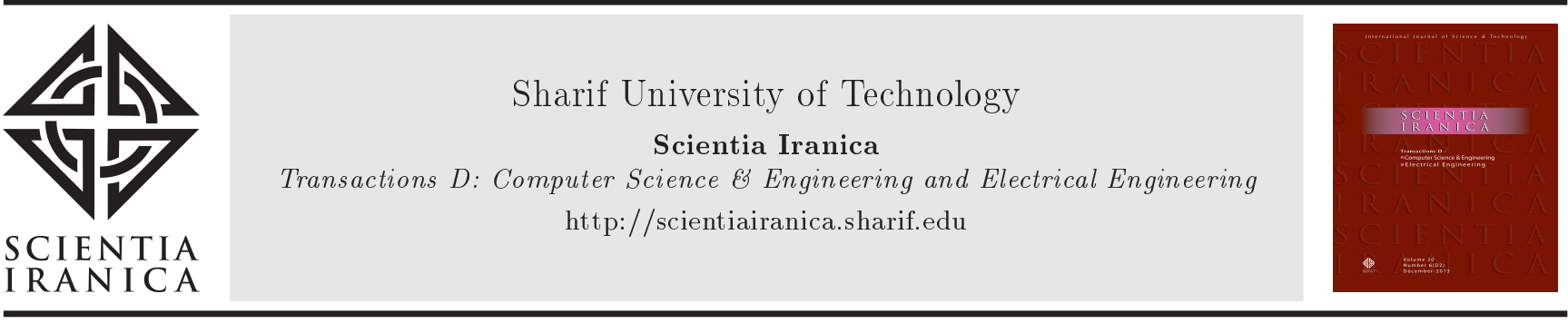

\title{
Design of alternating magnetic field generator for magnetic fluid hyperthermia research application
}

\author{
M. Mohseni and A. Rajaei* \\ Electrical and Electronics Engineering Department, Shiraz University of Technology, Modares Blvd., Shiraz, 71555-313, Iran.
}

Received 22 October 2015; received in revised form 24 September 2016; accepted 18 April 2017

\section{KEYWORDS}

AC magnetic field; Hyperthermia;

Resonant converter; Magnetic nanoparticles; solenoid coil.

\begin{abstract}
Hyperthermia utilizing nanoparticles is a novel cancer therapy, which relies on the heat released when nanoparticles inside a tumor are exposed to an alternating magnetic field. The field strength and frequency as the main variants affect performance of nanoparticles in heat generation. Besides characteristics of the nanoparticle, which are the main criteria for tuning amplitude and frequency of the magnetic field generated by an Alternating Magnetic Field Generator (AMFG), several related parameters should be considered for an optimum design of AMFG. These parameters are input voltage range, copper tube resistance used in solenoid coil, coil number of turns, etc. With regard to these criteria, a design procedure of AMFG for research applications is proposed to maximize the heat released by nanoparticles. In order to validate the design, an experimental setup of AMFG is prepared, which is used for in vivo hyperthermia tests. The experimental results are shown and compared with the simulations.

(C) 2018 Sharif University of Technology. All rights reserved.
\end{abstract}

\section{Introduction}

Magnetic nanoparticles (MNPs) have received significant interest in various fields of science. In biomedical applications, they have received considerable attention as a potential therapeutic tool for cancer and other diseases [1-5]. They are used as Magnetic Fluid Hyperthermia $(\mathrm{MFH})$ agents. Hyperthermia has long been regarded as a promising treatment by heating diseased tissues to between $43^{\circ} \mathrm{C}$ and $46^{\circ} \mathrm{C}$ for a period of time to destroy cancer cells without any lethal effect on normal tissues. MNPs are affected by an Alternating Magnetic Field (AMF), which leads to dissipation of thermal energy in the surrounding medium.

Several nanoparticle power loss measurement sys-

\footnotetext{
*. Corresponding author. Fax: +98 - 71- 37261288 E-mail addresses: mahdi.mohseni18@gmail.com ( $M$. Mohseni); a.rajaei@sutech.ac.ir (A.Rajaei)
}

tems have been presented in the literature [6-11]. The heating of the magnetic tracer embedded in a living tissue may be due to: the Brownian effect; eddy currents; fluctuations in the directions of the magnetic moments; crossing the anisotropic barrier of the tracer (Neel relaxation); or magnetic energy loss because of magnetic hysteresis loop [12-14]. Here, it is assumed that the heat dissipated by nanoparticles has its origin in hysteresis losses, which are proportional to the area of the hysteresis loop. Using Linear Response Theory (LRT), which is valid for low-amplitude fields, the heating power is given by [15-16]:

$$
P_{m}^{L R T}=\pi \mu_{0} x_{0} H_{0}^{2} f \frac{2 \pi f \tau}{1+(2 \pi f \tau)^{2}},
$$

where $x_{0}, H_{0}$, and $f$ are the equilibrium susceptibility, field amplitude, and field frequency, respectively. $\tau$ is the Néel-Brown relaxation time. Eq. (1) shows that electromagnetic field amplitude and frequency affect the dissipated power of MNP. 


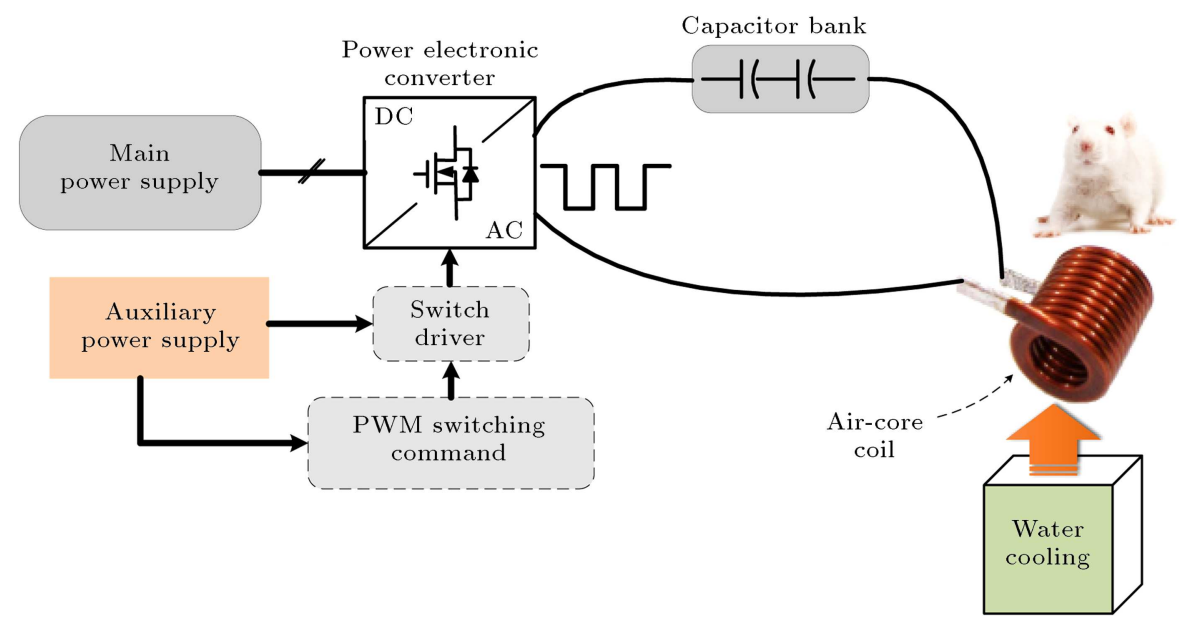

Figure 1. Schematic diagram of an AMF generator for hyperthermia application.

The maximum MNP power dissipation is desired as fast increase in temperature would improve the capability of the method to destroy cancer cells. Accordingly, Alternating Magnetic Field Generator (AMFG) should be designed (Figure 1).

Different systems of magnetic field generation have been proposed for magnetic nanoparticle hyperthermia experiments. AMFG using single-layer and double-layer solenoid, Helmholtz coils, and inductor with C-shaped ferromagnetic core are the most common systems, which have been compared in [17]. The most common type is single-layer solenoid [18-24] on which this study is focused.

Several studies have been conducted on AMFG improvement in hyperthermia application. In [18], the heating power of magnetic fluid, when exposed to a high-frequency magnetic field, was investigated. The setup was capable of generating homogeneous magnetic field of amplitudes up to $4 \mathrm{kA} / \mathrm{m}$ and frequencies from $10 \mathrm{kHz}$ to $1 \mathrm{MHz}$. The required analytical relationships and computations of power dissipation in magnetic fluid subjected to alternating magnetic field were developed in [19-20]. An AMFG operating at a frequency of $300 \mathrm{kHz}$ with variable field strength ranging from 0 to $11 \mathrm{mT}$ was designed in [21] and used for magnetic hyperthermia treatment.

Several investigations have been performed into high-frequency AMF measurement for the application of magnetic nanoparticle hyperthermia therapy. An alternating magnetic field measuring device was designed in [22], which used three detection coils as the probes and a DSP chip for data acquisition and processing. The intensity range of 18 Gauss to 78 Gauss and frequency range of $50 \mathrm{kHz}$ to $800 \mathrm{kHz}$ were considered for AMFG operation. Self-heating of thin wires required for accurate sensors used in AMF measurement was studied in [23].

In [24], alternating magnetic field optimization of iron oxide nanoparticles (IONP) in hyperthermia cancer treatment was described. As explained in [24], the production of eddy current heating is a function of tissue size, geometry, and composition as well as coil design and operation. The optimization was done by performing several experimental tests in different operating points and no analytical study was performed.

Analytical study of optimization of AMFG considering coil geometry and power electronic characteristics has not been performed so far. In this study, AMFG optimization steps regarding coil geometry and power electronic characteristics such as magnitude and frequency of voltage are described in detail.

Besides the above studies, which focused on the AMFG part of hyperthermia therapy, many studies have been conducted to develop analytical relationships and computations of power dissipation in magnetic fluid [2-11].

This paper explains the design procedure of AMFG for the purpose of maximizing the outcome of Eq. (1) while considering several practical constraints on power electronics resonant converter used to generate current through the solenoid coil. Therefore, the objective function is modified to achieve maximum performance of the system. Magnetic field frequency, coil number of turns and radius, and copper tube diameter are the main features of AMFG determined here.

This paper is organized as follows: The resonance converter and basic relations for the converter and solenoid coil are described in Section 2. Section 3 provides a review of design criteria and describes design optimization in detail. The results of simulations and experiments for the prepared system are presented and discussed in Section 4.

\section{Configurations, specifications, and basic operational principles of AMFG}

As shown in Figure 1, two main parts of an AMFG are as follows: 


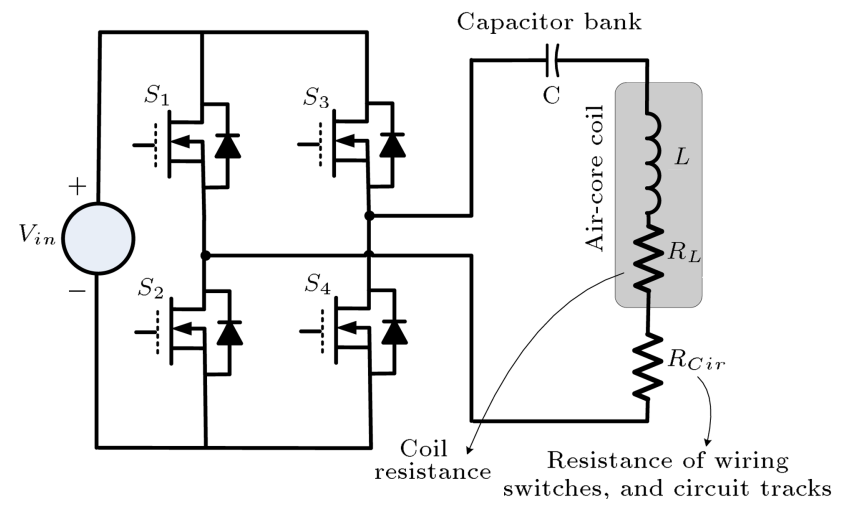

Figure 2. Basic structure of full-bridge ZCS series converter used as AMFG.

1. Power electronic resonant converter;

2. Air-core coil, which is the inductor part of resonant tank of converter $[25,26]$.

In order to inject high frequency-high amplitude alternating current into the air-core solenoid, several types of resonant converters are applicable. The main topologies are full-bridge Zero-Voltage Switching (ZVS), half-bridge ZVS, full-bridge Zero-Current Switching (ZCS), and half-bridge ZCS series converters. Regarding the voltage and current stress of power semiconductor switches, the appropriate structure can be selected. Accordingly, the most popular converter, which is also considered in this paper, is full-bridge ZCS series converter (Figure 2).

The converter should operate at resonant frequency to achieve maximum performance. Considering the basic operation of full-bridge ZCS series converter, resonance frequency $\left(f_{0}\right)$, peak value of current through the coil $\left(I_{M}\right)$, and peak value of voltage across coil at operating frequency of $f$ are as follows [27]:

$$
\begin{aligned}
f_{0} & =\frac{1}{2 \pi \sqrt{L C}}, \\
I_{M} & =\frac{2 V_{i n}}{\pi R_{T} \sqrt{1+Q_{L}^{2}\left(f / f_{0}-f_{0 / f}\right)^{2}}}, \\
V_{M} & =2 \pi f L I_{M},
\end{aligned}
$$

where, $R_{T}$ is the total resistance of the circuit $\left(R_{T}=\right.$ $R+R_{L}$ ) and $Q_{L}$ is the loaded quality factor defined as:

$$
Q_{L}=\frac{\sqrt{L / C}}{R_{T}} .
$$

Power consumption of the circuit is equal to power loss of $R_{T}$.

$$
P_{\text {in }}=\frac{1}{2} R_{T} I_{M}^{2}
$$

As mentioned before, the second basic part of AMFG is air core coil. In the design calculations, three parameters of coil are used: electromagnetic field generated by coil $(B)$, coil inductance, and coil resistance $\left(R_{L}\right)$. Peak value of sinusoidal $B\left(B_{M}\right)$ generated in an air core coil and inductance of a singlelayer air core coil $(L)$ are given by:

$$
\begin{aligned}
& B_{M}=\frac{\mu_{0} N I_{M}}{l}, \\
& L=\frac{0.001 * N^{2} r^{2}}{\left(228 r^{2}+254 l\right)},
\end{aligned}
$$

where, $N, r$, and $l$ are number of turns, coil radius, and coil length, respectively. Considering skin effect in high-frequency operation, coil resistance is also calculated as:

$$
\begin{aligned}
& R_{L}=2 \pi r N \frac{\rho}{\pi(D-\delta) \delta}, \\
& \delta \approx \sqrt{\frac{\rho}{\pi f \mu_{0}}},
\end{aligned}
$$

where, $\rho$ is relative resistance and equal to $1.68 \times 10^{-8}$ for copper, and $D$ is the diameter of tube used to make the coil.

\section{Design optimization of AMFG}

The aim of design procedure is to maximize the generated heat by nano particles according to Eq. (1). Using Eq. (1) and Eqs. (6)-(10), the relation for heat generation is as follows:

$$
\begin{aligned}
& P_{m}^{L R T}=\left(\frac{\pi x_{0} \mu_{0}^{2}}{D^{2}}\right)\left(\frac{P_{i n}(D-\delta) \delta}{\rho N r}\right)\left(\frac{(2 \pi \tau) f_{0}^{2}}{1+(2 \pi \tau)^{2} f_{0}^{2}}\right) \\
& \delta=\sqrt{\frac{\rho}{\pi \mu_{0} f_{0}}} .
\end{aligned}
$$

It is assumed that system input power, which is approximately equal to the power consumed by total system resistance, is constant.

In the following, some practical limitations of the system which should be considered in the design procedure are described:

A) Inability to work exactly at resonant frequency. One of the main practical constraints which should be considered is that tuning the operating frequency at resonant frequency is not possible. This is mainly because:

1) Inserting the samples into the coil changes the coil inductance and resistance, hence, changing resonant frequency; 


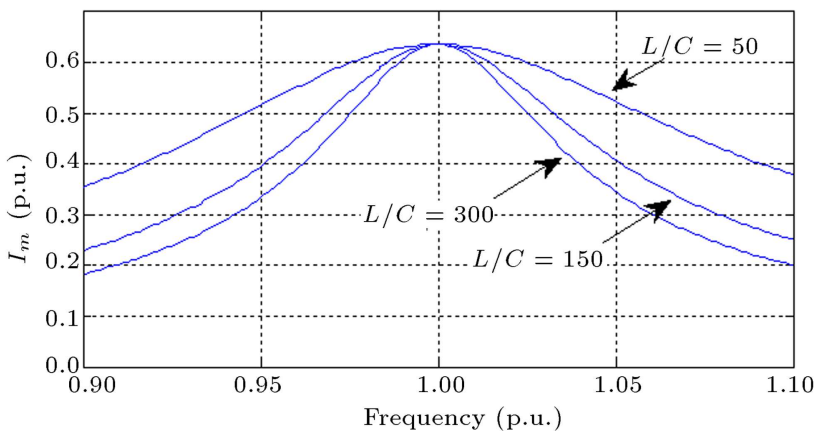

Figure 3. $I_{M}$ variation for a given resonant frequency $(L * C=C N S T)$ and different values of $L / C$. $V_{\text {in }}=1$ p.u., $R_{T}=1$ p.u., $f_{0}=1$ p.u.

2) Circuit wiring changes the total inductance of circuit; and

3) The system is temperature-dependent.

Hence, to achieve maximum performance at a given frequency, which means achieving maximum current, operating at a frequency around the resonant frequency should be considered. Therefore, the best system performance is when the variation of $I_{M}$ is minimum while system frequency deviates from resonant frequency. As shown in Figure 3, for a given resonant frequency, decreasing value of $L$ results in lower $I_{M}$ variation.

Therefore, besides maximizing Eq. (1), small value of $L$ is desirable.

B) Dead time. Another practical constraint of system operation is dead time, which should be applied to switching commands to prevent shooting through the legs. To prevent cross conduction, two switches of a leg (S1 and S2, S3 and S4) should not be on simultaneously; therefore, the command waveforms should be non-overlapping and have sufficient dead time. Because of MOSFET delay time at turn-off, if the dead time is too short, one switch would still remain on while the other turns on. This results in cross conduction current pulses $\left(I_{p k}=V_{i n} /\left(R_{D S 1}+R_{D S 2}\right)\right)$, which may cause immediate failure of the devices.

Considering dead time for the switching command, the peak current through the resonant tank decreases as the effective square wave voltage applied to it decreases. The modified amplitude of the generated current by resonant converter is given by:

$$
I_{M}=\frac{2 V_{i n}\left(1-T_{d} f_{0}\right)}{\pi R_{T} \sqrt{1+Q_{L}{ }^{2}\left(f_{/} f_{0}-f_{0 / f}\right)^{2}}},
$$

where $T_{d}$ is the dead time interval.

C) Desirability of lower input dc voltage. Very small value of switch resistance is highly desirable, not only to reduce power loss of the system but also to limit thermal power dissipated from switches. It simplifies thermal management and affect the reliability of converter. On the other hand, onresistance of power MOSFET rapidly increases with rated blocking voltage; therefore, working at low dc voltage is important and should be considered in design optimization.

It is worth to pay attention to some points:

1) As mentioned before, the input power of the system is assumed to be constant. Obviously, higher input power improves the system performance.

2) Coil length can be calculated as:

$$
l=k N D,
$$

where $k$ is the coefficient used to show insulating cover of tube. Here, it is assumed to be equal to 1.1. Therefore, coil length is not an independent variable in the calculations.

3) For in vivo research application, coil radius should be at least $2 \mathrm{~cm}$.

4) System resistance $\left(R_{T}\right)$ contains two main parts. First part includes the wiring resistance on $\mathrm{AC}$ side and coil resistance $\left(R_{L}\right)$ which varies depending on system frequency (due to skin effect), and the second part includes converter switch on-state, wiring resistance on DC side, and track resistance $\left(R_{C i r}\right)$ which are rather constant.

$$
R_{T}=R_{C i r}+R_{L}
$$

The parameters of converter and coil which should be determined in the design procedure are as follows:

1) Resonant frequency $\left(f_{0}\right)$;

2) Number of turns $(N)$;

3) Tube diameter $(D)$;

4) Coil radius $(r)$.

According to the above discussion, the objective function which should be minimized to achieve best performance is:

$$
O F=\frac{1}{P_{m}^{L R T}\left(N, r, D, f_{0}\right)}+k_{1} L_{(N, r, D)}^{2} f_{0}^{2}+k_{2} V_{i n},
$$

where, $L$ is obtained by Eq. (8) and $P_{m}^{L R T}$ and $V_{i n}$ are calculated as:

$$
\begin{aligned}
P_{m}^{L R T}{ }_{\left(N, r, D, f_{0}\right)}=\left(\pi x_{0} \mu_{0}\right)\left(\frac{1}{1.1 D}\right)^{2} \\
\\
\quad\left[\frac{8\left(1-T_{d} f_{0}\right)^{2} P_{i n}^{2}}{\pi^{2}\left(R_{C i r}+R_{L}\right)}\right]\left(\frac{(2 \pi \tau) f_{0}{ }^{2}}{1+(2 \pi \tau)^{2} f_{0}{ }^{2}}\right),
\end{aligned}
$$




$$
V_{i n}=\sqrt{\frac{\pi}{2}\left(R_{C i r}+R_{L}\right) P_{i n}}
$$

$k_{1}$ and $k_{2}$ are weight coefficients.

D) The genetic algorithm (GA). The genetic algorithm (GA) used here is described in the following.

\subsection{Optimization method}

GAs are search and optimization methods based on natural evolution. They can search possible solutions simultaneously and do not need any previous knowledge or special properties of the fitness function. They consist in a population of strings (chromosomes) transformed by three genetic operators, namely, selection, crossover, and mutation. Each chromosome represents a possible solution to the problem which is being optimized and each bit represents a value for some variable of the problem (gene). These solutions are classified by a fitness function in order to obtain better solutions [28].

\subsection{Encoding}

The objective is to find the optimal number of turns, diameter of coil, frequency, and input voltage parameters to maximize heating power of device. Therefore, the configuration of population is encoded by two parameters, namely, frequency and input voltage. The first value of resonance frequency parameter is equal to $100 \mathrm{kHz}$ and the second parameter, i.e., input voltage starts from $5 \mathrm{v}$.

\subsection{Fitness function}

The objective function will be evaluated for each individual of the population. The fitness is a measure of quality, which is used to compare different solutions. In this study, the fitness is defined as Eq. (15).

The GAs only find the minimum positive value of the objective function and, to do so, they will find optimal frequency and input voltage of the device and maximize heating power.

\subsection{Initial population}

An initial population, called parents, consisting of $N$ sequences of chromosomes is randomly generated. The number of initial population is chosen by designer. Increasing initial population increases probability of finding global minimum and decreases speed of algorithm due to additional calculations. The regenerated chromosomes are called offspring. The regeneration results in chromosomes with better fitness values. The algorithm proceeds until no more improvement is attained in fitness function.

\subsection{Crossover}

The main objective of crossover is to rearrange the information of two different chromosomes and produce a new one. Crossover section has two steps. First, chromosomes are chosen randomly and, then, every chosen chromosome can be recombined by probability of $P_{c}\left(0 \leq P_{c} \leq 1\right)$.

\subsection{Mutation}

An inherent disadvantage of the crossover is that at some particular position, the value of the gene may not change at all. To avoid this problem, mutation is used to introduce some arrangement of artificial diversification in the population to avoid premature convergence on local optimum. In this method, one or more genes of the chosen sequence are changed by probability of $P_{m}\left(0 \leq P_{m} \leq 1\right)$ and a new sequence is generated. Mutation operator is applied at each part of the chromosome, separately.

\section{Simulation and experimental results}

In order to prepare AMFG setup with $600 \mathrm{~W}$ input power, the objective function presented in Section 3 is minimized using Genetic Algorithm (GA). GAs are search and optimization methods based on natural evolution. They can search possible solutions simultaneously and do not need any previous knowledge or special properties of the fitness function. They consist in a population of strings (chromosomes) transformed by three genetic operators, namely, selection, crossover, and mutation. Each chromosome represents a possible solution to the problem which is being optimized and each bit represents a value for some variable of the problem (gene).

Power MOSFET is used to make the setup and the characteristics (Table 1) are considered for modeling and simulations in IRF1404 [29]. Two MOSFETs are parallel in each switch to decrease the total on-state switch resistance $\left(R_{D S}\right)$ and, therefore, the related power loss. Because of the characteristics of power MOSFET and gate driver, a constant dead time of 250 nsec. is applied to the switching signals of two switches in a leg (Figure 4).

Regarding the required characteristics of the system, it is necessary to apply an ultrafast gate drive circuit to the MOSFETs. IXDD414 is used as the gate

Table 1. Power MOSFET characteristics applied in simulations and driver circuit design of the prepared setup.

\begin{tabular}{lll}
\hline Symbols & \multicolumn{1}{c}{ Definitions } & \multicolumn{1}{c}{ Values } \\
\hline$V_{B R}$ & Drain to source breakdown voltage & $40(\mathrm{~V})$ \\
$I_{D}$ & Continuous drain current & $121(\mathrm{~A})$ \\
$R_{D S}$ & Static drain to source on-resistance & $4(\mathrm{~m} \Omega)$ \\
$Q_{G}$ & Total gate charge $\left(V_{G S}=10 \mathrm{~V}\right)$ & $196(\mathrm{nC})$ \\
$t_{d(\text { on })}$ & Turn-on delay time & $17(\mathrm{nsec})$ \\
$t_{d(\text { off })}$ & Turn-off delay time & $46(\mathrm{nsec})$ \\
$t_{r}$ & Rise time & $190(\mathrm{nsec})$ \\
$t_{\text {off }}$ & Fall time & $33(\mathrm{nsec})$ \\
\hline
\end{tabular}




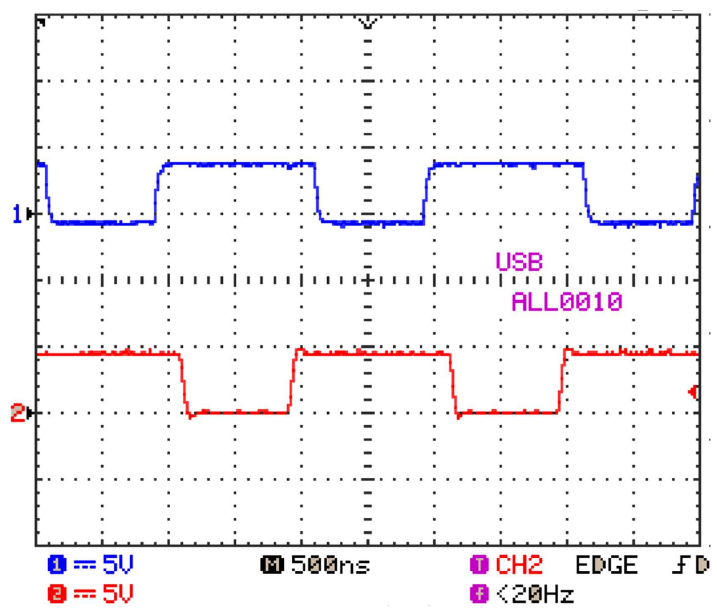

(a)

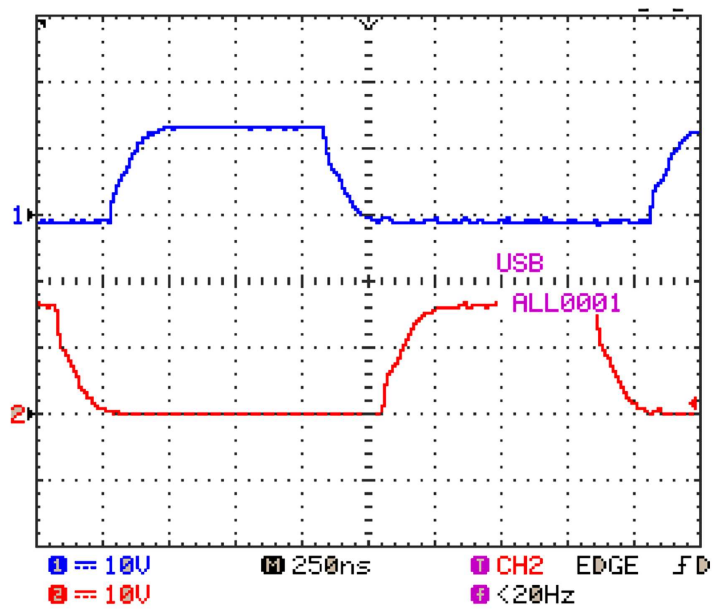

(b)

Figure 4. Gate drive signals of two switches in a leg while considering a dead time of 250 nsec: (a) Control signals applied to the gate drive circuit, and (b) applied voltage to the gate of MOSFET (250 nsec./div., $10 \mathrm{~V} /$ div.).

drive of MOSFETs, which is able to deliver $14 \mathrm{~A}$ at the output [30].

Also, $\boldsymbol{\tau}$ and $x_{0}$ are assumed to have typical values of $9 \times 10^{-9}$ and $10^{-8}$, respectively [31]. It should be noted that $D$ is considered to have a constant value of $8 \mathrm{~mm}$ in the design optimization algorithm, as it was the only available size of copper tube to make the coil. According to the data acquired by the design procedure, an experimental setup is prepared (Figure 5) and equivalent circuit is simulated to validate the used relations of the system of which the results are shown in Table 2.

The second version of AMFG prepared for in vivo hyperthermia research is shown in Figure 6.

The AMFG is simulated, in which the injected current of solenoid as well as solenoid and capacitor voltages is shown in Figure 7. The experimental results of AMFG for output voltage of $\mathrm{H}$-bridge converter and coil voltage are shown in Figure 8.

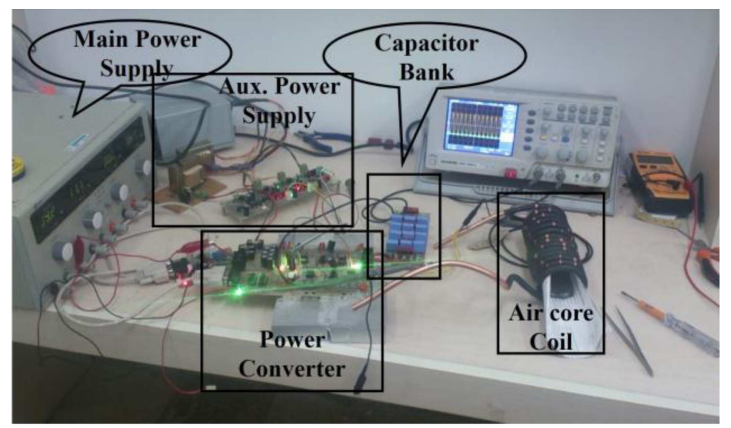

(a)

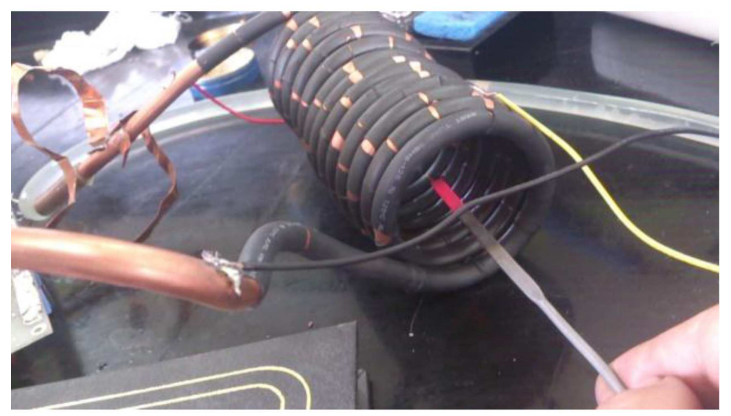

(b)

Figure 5. Experimental setup: (a) Setup and (b) solenoid coil.

Table 2. Characteristics of experimental setup with respect to presented design procedure.

\begin{tabular}{lll}
\hline Symbols & \multicolumn{1}{c}{ Definitions } & \multicolumn{1}{c}{ Values } \\
\hline$P_{i n}$ & Input power of AMFG & $600(\mathrm{~W})$ \\
$V_{i n}$ & Input voltage of AMFG & $22(\mathrm{~V})$ \\
$C$ & Capacitance of capacitor tank & $80(\mathrm{nF})$ \\
$N$ & Number of turns & 15.4 \\
$D$ & Diameter of copper tube & $8(\mathrm{~mm})$ \\
$r$ & Coil radius & $2.5(\mathrm{~cm})$ \\
$l$ & Coil length & $13(\mathrm{~cm})$ \\
$L$ & Inductance of air-core coil & $4.6(\mu \mathrm{H})$ \\
& Peak value of electromagnetic & $0.011(\mathrm{~T})$ \\
$B_{M}$ & field & \\
& & $250(\mathrm{nsec})$ \\
$T_{d}$ & Dead time & \\
& On-state MOSFET and circuit & $0.05(\Omega)$ \\
$R_{C i r}$ & resistance & \\
& & \\
$I_{M}$ & Peak value of current through & $112(\mathrm{~A})$ \\
& coil & $75(\mathrm{~mW})$ \\
$P_{m}^{L R T}$ & Hysteresis Power & $507216(\mathrm{~Hz})$ \\
$f_{0}$ & Resonant switching frequency
\end{tabular}

It should be mentioned that as high-voltage probe was not available for measurement, the voltage across a part of the coil was measured. Using the measured voltage, the voltage across the coil could be easily determined by a correction factor of 5.45 (Figure 9 ). 


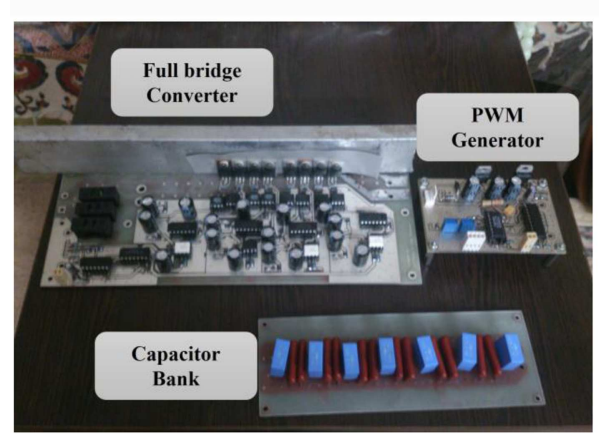

(a)

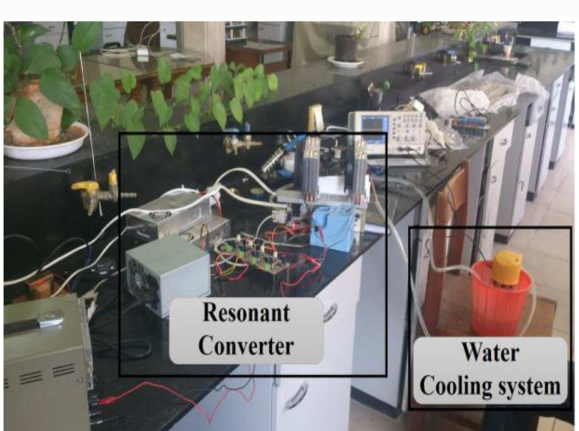

(b)

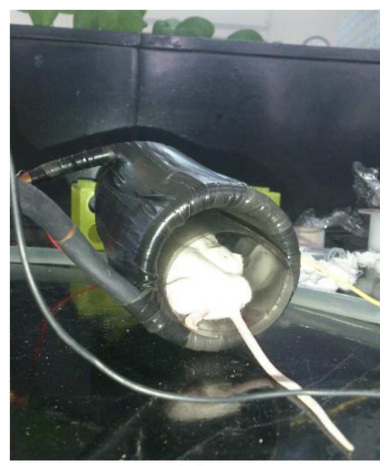

(c)

Figure 6. AMFG setup prepared for in vivo hyperthermia research: (a) Three main parts of the system, (b) setup, and (c) solenoid coil during in vivo test.

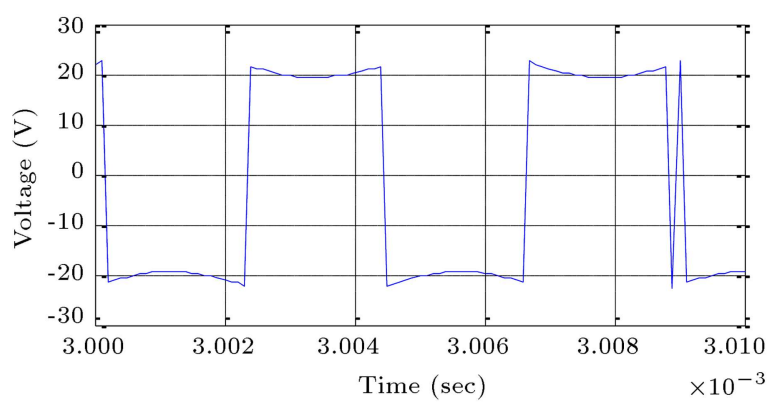

(a)

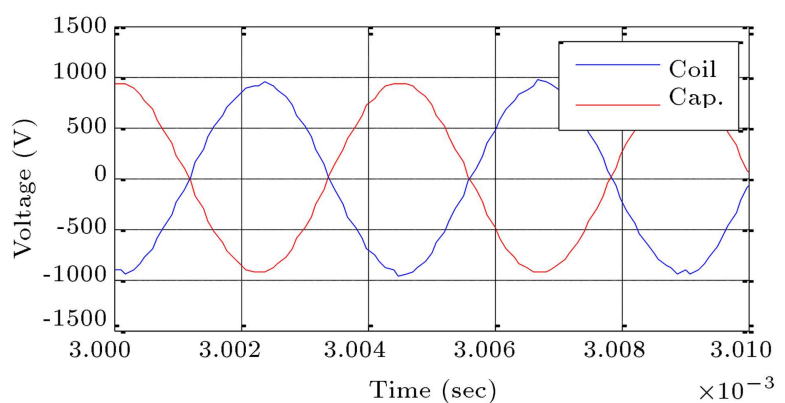

(b)

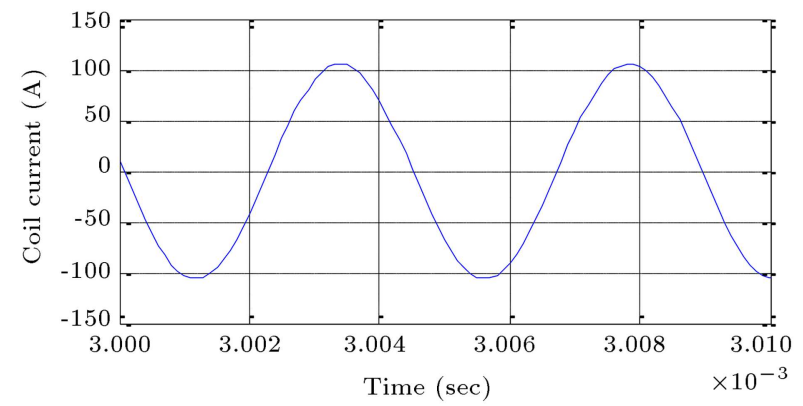

(c)

Figure 7. Simulation Results: (a) Square voltage applied to resonant tank, (b) capacitor and coil voltages, and (c) coil current.

In order to investigate the performance of the optimized system, the AMFG with two different coils is implemented. The operating power, input voltage, and switching frequency considered for the tests are the same and equal to $600 \mathrm{~W}, 22$ Volts, and $500 \mathrm{kHz}$,

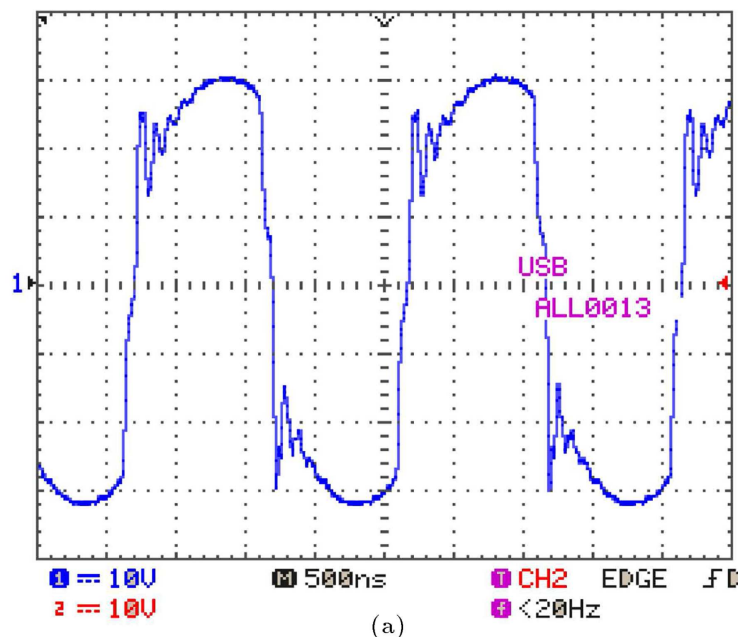

(a)

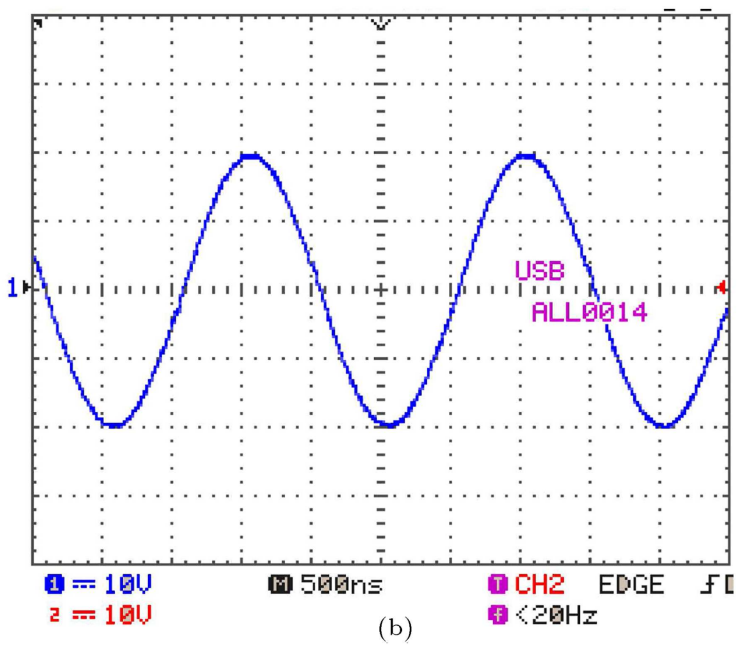

Figure 8. Experimental results: (a) Square wave voltage applied to resonant tank ( $10 \mathrm{~V} /$ div., $500 \mathrm{nsec} . /$ div.), and (b) solenoid coil voltage (545 V/div., 500 nsec./div.).

respectively. The geometry of the coils is as follows:

- Optimized coil: diameter: $5 \mathrm{~cm}$, number of turns: 15, length: $13 \mathrm{~cm}$ (Figure 10).

- Non-Optimized coil: diameter: $9 \mathrm{~cm}$, number of turns: 16, length: $16 \mathrm{~cm}$ (Figure 11). 


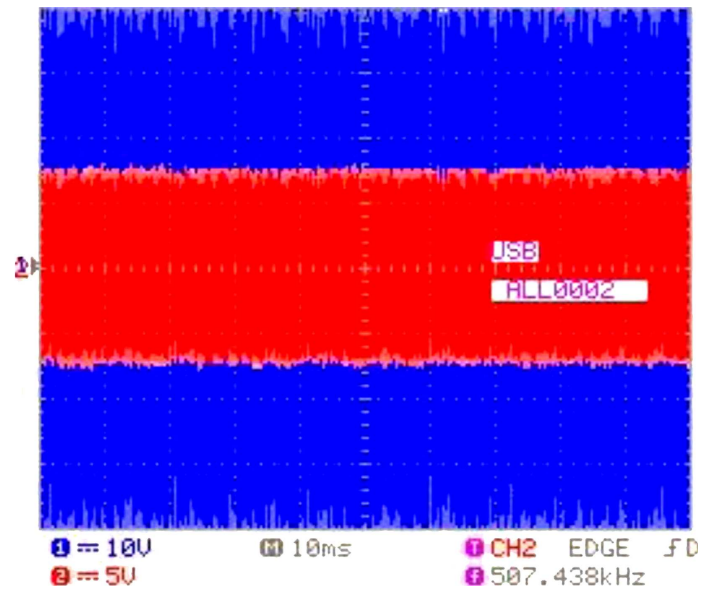

Figure 9. Measuring coil voltage: blue: voltage across coil (10 V/div., $10 \mathrm{msec} . /$ div.), and red: voltage across part of coil (5 V/div., $10 \mathrm{msec} . /$ div. $)$.

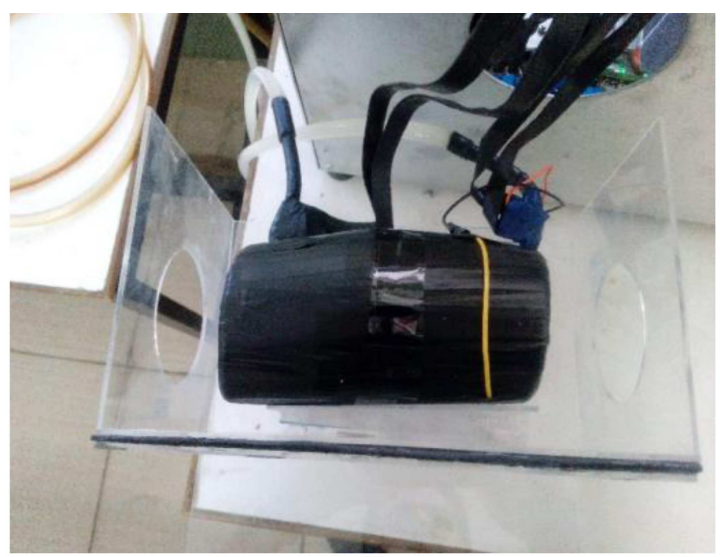

(a)

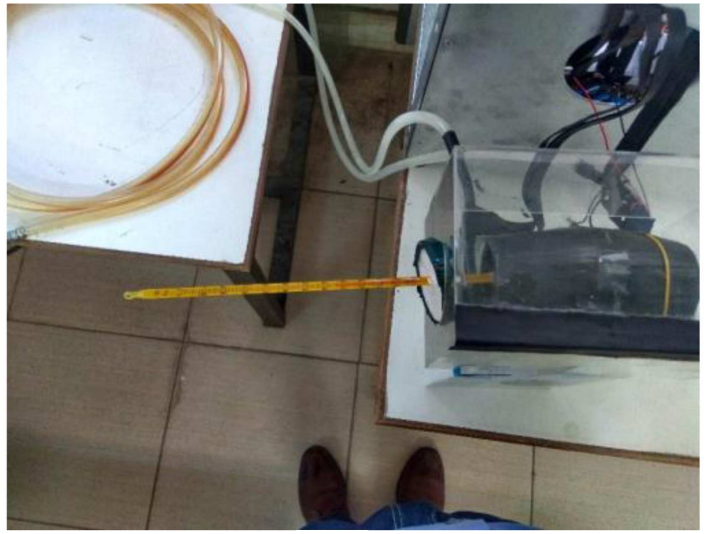

(b)

Figure 10. Experimental setup while using optimized coil: (a) Optimized coil and (b) test with magnetic nano-particles.

The experimental tests are done with the same magnetic nano-particle (doze: $6 \mathrm{mg}, 4$ milliliters, custom-made magnetic ferrofluid form) and the duration of both tests is 420 seconds. The results are shown in Table 3 and Figure 12. As it can be seen,

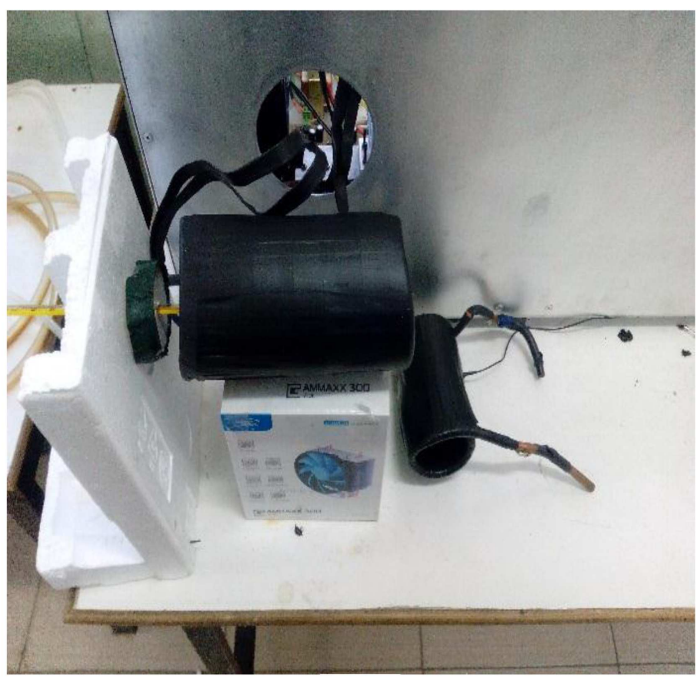

(a)

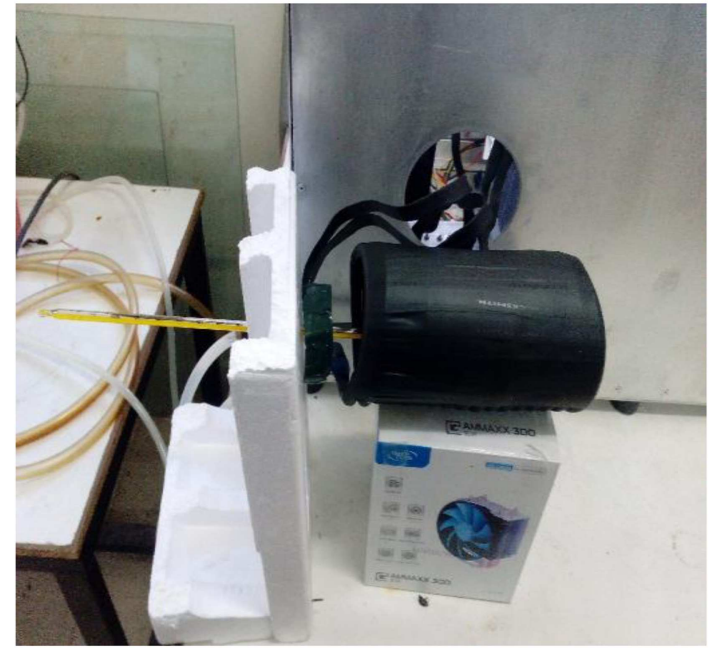

(b)

Figure 11. Experimental setup while using non-optimized coil: (a) Non-optimized coil compared with optimized coil and (b) test with magnetic nano-particles.

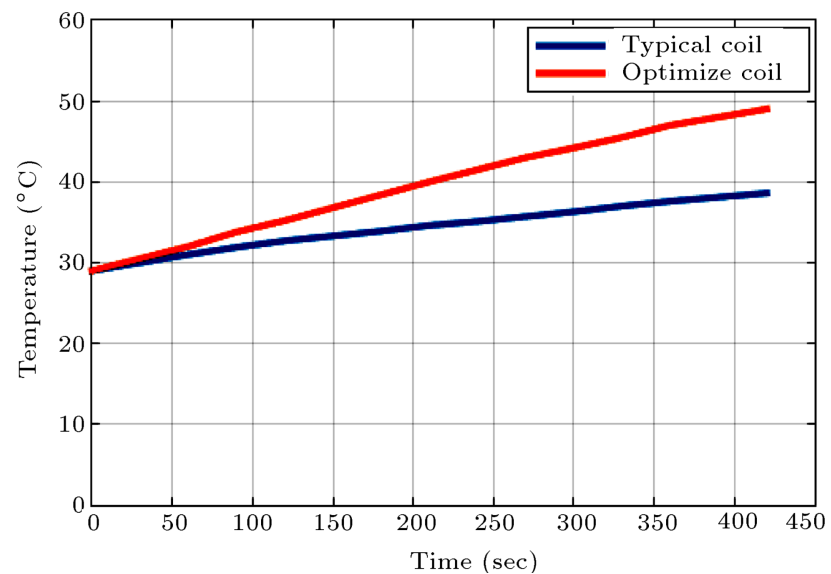

Figure 12. Experimental result of temperature rise for magnetic nano-particles while using optimized and non-optimized coils. 
Table 3. Experimental results of temperature rise for optimized and non-optimized coils.

\begin{tabular}{ccc}
\hline & \multicolumn{2}{c}{ Temperature $\left({ }^{\circ} \mathbf{C}\right)$} \\
\cline { 2 - 3 } Time (sec) & $\begin{array}{c}\text { Optimized } \\
\text { coil }\end{array}$ & $\begin{array}{c}\text { Non-optimized } \\
\text { coil }\end{array}$ \\
\hline 0 & 29 & 29 \\
30 & 30 & 30.5 \\
60 & 31 & 32 \\
90 & 31.9 & 33.8 \\
120 & 32.7 & 35.2 \\
150 & 33.3 & 36.8 \\
180 & 33.9 & 38.4 \\
210 & 34.6 & 40 \\
240 & 35.1 & 41.5 \\
270 & 35.7 & 43 \\
300 & 36.3 & 44.2 \\
330 & 37 & 45.5 \\
360 & 37.6 & 47 \\
390 & 38.1 & 48 \\
420 & 38.6 & 49 \\
\hline
\end{tabular}

temperature rise of magnetic nano-particles is more while using optimized coil, which shows higher heat generation and demonstrates analytical design of the system.

\section{Conclusion}

In this paper, design optimization of AMFG for hyperthermia research applications was described in detail and various practical constraints were considered. The main constraints were inability to work in resonant frequency, requiring dead time for generating switching command signals, and minimizing switch conduction power loss by working at low input dc voltage. An objective function for maximizing dissipated power by nano particles was derived, which considered the above-mentioned constraints. Using genetic algorithm, optimized parameters of the system for a $600 \mathrm{~W}$ AMFG were calculated. These parameters were resonant frequency, copper tube diameter, coil number of turns, and coil radius. According to the presented design procedure, the AMFG was prepared and used for in vivo hyperthermia research application.

\section{References}

1. Kunisaki, J., Saito, T., Morita, M., Yamada, T., and Takemura, Y. "Temperature rise of resonant circuits for hyperthermia excited by weak RF magnetic field", IEEE Int. Magnetics Conf. INTERMAG'06., San Diego, California, USA (2006).

2. Wust, P., Hildebrandt, B., and Sreenivasa, G. "Hyper- thermia in combined treatment of cancer", The Lancet Oncology, 3(8), pp. 487-497 (2002).

3. Falk, M. and Issels, R. "Hyperthermia in oncology", Int. Jour. of Hyperthermia, 17(1), pp. 1-18 (2001).

4. Chang, E., Alexander, H., and Libutti, S. "Laparoscopic continuous hyperthermic peritoneal perfusion", $J$. of the American College of Surgeons, 193(2), pp. 225-229 (2001).

5. Tasci, T., Vargel, I., Arat, A., Guzel, E., Korkusuz, P., and Atalar, E. "Focused RF hyperthermia using magnetic fluids", Med. Phys., 36(5), pp. 1906-1912 (2009).

6. Glöckl, G., Hergt, R., Zeisberger, M., Dutz, S., Nagel, S., and Weitschies, W. "The effect of field parameters, nanoparticle properties and immobilization on the specific heating power in magnetic particle hyperthermia", J. Phys.: Condens. Matter, 18(38), pp. 2935-2949 (2006).

7. Natividad, E., Castro, M., and Mediano, A. "Accurate measurement of the specific absorption rate using a suitable adiabatic magnetothermal setup", Appl. Phys. Lett., 92(9), pp. 093116-093116-3 (2008).

8. Natividad, E., Castro, M., and Mediano, A. "Adiabatic vs. non-adiabatic determination of specific absorbtion rate of ferrofluids", J. Magn. Magn. Mater., 321(10), pp. 1497-1500 (2009).

9. Atsumi, T., Jeyadevan, B., Sato, Y., and Tohji, K. "Heating efficiency of magnetite particles exposed to AC magnetic field", J. Magn. Magn. Mater., 310(2), pp. 2841-2843 (2007).

10. Eggeman, A., Majetich, S., Farrell, D., and Pankhurst, Q. "Size and concentration effects on high frequency hysteresis of iron oxide nanoparticles", IEEE Trans. Magn., 43(6), pp. 2451-2453 (2007).

11. Rosensweig, R. "Heating magnetic fluid with alternating magnetic field", J. Magn. Magn. Mater., 252, pp. 370-374 (2002).

12. Kuznetsov, A., Shlyakhtin, O., Brusentsov, N., and Kuznetsov, O. "Smart mediators for self-controlled inductive heating", European Cells and Materials, 3(2), pp. 75-77 (2002).

13. Nedelcu, G. "The heating study of two types of colloids with magnetite nanoparticles for tumours therapy", Digest J. of Nanomaterials and Biostructures, 3(2), pp. 99-102 (2008).

14. Zhao, M., Hun, J., Zou, J., Zhao, B., and Li. Y. "Characteristics of a magnetic fluid under an orthogonal alternating magnetic field", J. Magn. Magn. Mater., 409, pp. 66-70 (2016).

15. Branquinho, L., Carri, M., Costa, A., Zufelato, N., Sousa, M., Sousa, R., Miotto, R., Ivkov, A., and Bakuzis, A. "Effect of magnetic dipolar interactions on nanoparticle heating efficiency: Implications for cancer hyperthermia", Scientific Reports, 3, pp. 1-10 (2013).

16. Khandhar, A., Ferguson, R., Simon, J., and Krishnan, K. "Enhancing cancer therapeutics using sizeoptimized magnetic fluid hyperthermia", J. Appl. Phys., 111(7), pp. 7B306-7B3063 (2012). 
17. Skumiel, A., Leszczyński, B., Molcan, M., and Timko, M. "The comparison of magnetic circuits used in magnetic hyperthermia", J. Magn. Magn. Mater., 420, pp. 177-184 (2016).

18. Bekovic, M. and Hamler, A. "Determination of the heating effect of magnetic fluid in alternating magnetic field", IEEE Trans. on Magnetics, 46(2), pp. 552-555 (2010).

19. Rosensweig, R. "Heating magnetic fluid with alternating magnetic field", J. Magn. Magn. Mater., 252, pp. 370-374 (2002).

20. Carrey, J., Mehdaoui, B., and Respaud, M. "Simple models for dynamic hysteresis loop calculations of magnetic single-domain nanoparticles: application to magnetic hyperthermia optimization", J. Appl. Phys., 109(8), pp. 083921-17 (2011).

21. Wang, X., Chen, Y., Huang, C., Wang, X., Zhao, L., Zhang, X., and Tang, J. "Contribution of a 300 $\mathrm{kHz}$ alternating magnetic field on magnetic hyperthermia treatment of HepG2 cells", Bio Electromagnetics, 34(2), pp. 95-103 (2013).

22. Wu, J., Cai, D., Cao, X., and Tang, J. "A novel alternating magnetic field measuring device for magnetic induction hyperthermia", Int. Conf. on Complex Medical Engineering, Beijing, China (2013).

23. Boekelheide, Z., Hussein, Z., and Hartzell, S. "Electronic measurements in an alternating magnetic field (AMF) for studying magnetic nanoparticle hyperthermia: Minimizing eddy current heating", IEEE Trans. on Magnetics, 52(7), pp. 1-4 (2016).

24. Kastnera, E., Reevesb, R., Bennetta, W., Misraa, A., Petrykb, J., Petryka, A., and Hoopesb, P. "Alternating magnetic field optimization for IONP hyperthermia cancer treatment", Proc. of SPIE Conf., San Francisco, California, USA (2015).

25. Nemkov, V., Ruffini, R., Goldstein, R., Jackowski, J., DeWeese, T., and Ivkov, R. "Magnetic field generating inductor for cancer hyperthermia research", The Int. $J$. for Computation and Mathematics in Elec. and Electronic Eng., 30(5), pp. 1626-1636 (2011).
26. Bordelon, D., Goldstein, R., Nemkov, V., Kumar, A., Jackowski, J., DeWeese, T., and Ivkov, R. "Modified solenoid coil that efficiently produces high amplitude AC magnetic fields with enhanced uniformity for biomedical applications", IEEE Trans. Magnetics, 48(1), pp. 47-52 (2012).

27. Kazimierczuk, M. and Czarkowski, D., Resonant Power Converters, 2nd Edn., Wiley-IEEE Press (2011).

28. Salehinia, A., Haghifam, M., Shahabi, M., and Mahdloo, F. "Energy loss reduction in distribution systems using GA-based optimal allocation of fixed and switched capacitors", 2010 IEEE International Energy Conference, Manama, pp. 835-840 (2010).

29. International Rectifier IR, IR1404 Datasheet, IR Corporation, CA (2003).

30. IXYS Low-Side Ultrafast MOSFET Driver, IXDD414 datasheet, IXYS Corporation, CA (2004).

31. Raikher, Y. and Stepanov, V. "Linear and cubic dynamic susceptibilities of superparamagnetic fine particles", Phys. Rev. B, 55(22), pp. 15005-15017 (1997).

\section{Biographies}

Mahdi Mohseni was born in Lamerd, Iran. $\mathrm{He}$ received his BSc degree in Electrical Engineering from Birjand University, Birjand, Iran. He is currently MSc student at Shiraz University of Technology, Shiraz, Iran. His main research interests are power electronics, resonant converters, and magnetic field analysis.

Amirhossein Rajaei was born in Jahrom, Iran. He received MS and $\mathrm{PhD}$ degrees in Electrical Engineering from Tarbiat Modares University, Tehran, Iran, in 2009 and 2013, respectively. Since 2013, He is with Shiraz University of Technology, Shiraz, Iran, as assistant professor. His main research interests include renewable energy resources, power converters, electric vehicles, and motor drive systems. 